mRNA by anticodons on tRNA (one consequence of which is the suppression of nonsense codons). In $E$, coli cells carrying the ram gene, seven per cent of the cellular protein was degraded within 120 minutes, whereas in wild type cells only two per cent of the proteins was degraded in this time. Similarly increased rates of degradation were observed in strains of $E$. coli carrying mis-sense suppressor tRNAs, which only mistranslate the codons responded to by one tRNA molecule. Yet another test was to show that proteins containing analogues of aminoacids were much less stable than their wild type counterparts containing the proper amino-acids.

What is the basis for the action of the degradation system? Its general availability for prematurely terminated or mis-sequenced proteins implies that all normal $E$. coli proteins must share certain common features which mark them as inviolate from the action of whatever enzymes constitute the system. Deviations too far from these norms would make the proteins susceptible to proteolysis; small extents of mutation would presumably not matter. The common features are presumably concerned with the tertiary conformation of the protein, although this does raise the question of why incomplete proteins still under synthesis are not degraded; per. haps they are protected by the ribosome. One important experiment would be to detect mutants in the degradation system, but it is difficult to think of suitable selective tests. A particularly intriguing question is whether similar systems in other bacteria would recognize as normal similar features of tertiary structure, or whether each bacterial species has its own standards.

\section{BACTERIA \\ Slimmed Down Phage}

from our Cell Biology Correspondent

ON occasion and perhaps more often than might be anticipated, bacteria can, it seems, turn the tables on a virulent bacteriophage and render it into a bacteriocin, a defective phage particle unable even at very high multiplicities of infection to lyse its host cell but which can adsorb to and kill other sensitive bacteria. The colicins, bacteriocins of strains of Escherichia coli, needless to say, have been more extensively studied than their counterparts in the more arcane bacterial genera, but fortunately not everybody is wedded to $E$. coli. $R$ cently, for example, in the Journal of Virology $(9,160 ; 1972)$ Lotz and Mayer have described an exemplary investigation of a bacteriocin produced by a strain of Rhizobium lupini isolated from the root nodules of a lupin. Al- though lupins are not of very great economic importance, other legumes which harbour species of Rhizobium are extremely important crop plants and anything that furthers knowledge and understanding of bacteria of the genus Rhizobium is valuable.

It turns out that one of the strains of Rhizobium lupini, known as 16-3, produces a substance of high molecular weight that not only inhibits the growth of a second strain, designated $16-2$, but also affects the replication of a lytic phage in strain 16-2. The bacteriocin, when added early during the latent period of the phage in 16-2 cells, inhibits their lysis whereas when added late in the latent period it accelerates lysis. Under the electron microscope samples of the bacteriocin can be seen to contain large numbers of particles which, with a sheath and core structure terminated by a base plate and spikes, closely resemble the tails of, for example, T-even coliphages.

When sucrose gradients of the bacteriocin preparations are assayed for bactericidal activity, the single peak of this activity corresponds with a small optical density peak and material in this fraction is seen in the electron microscope to consist exclusively of phage tail structures. As anticipated these tails adsorb to sensitive 16-2 strain cells and the sheath contracts on adsorption. Because the bactericidal activity resists inactivation by doses of ultraviolet radiation as high as $6,000 \mathrm{ergs} / \mathrm{mm}^{2}$ it seems unlikely that the phage tails contain any nucleic acid and it is much more likely that the tails kill sensitive cells either by injecting lytic enzymes or by acting at the cell surface. But in any event it seems clear that the bacteriocin of $R$. lupini strain $16-3$ is an incomplete phage particle.

Delk and Dekker (J. Mol. Biol., 64, 287 ; 1972) reach what is essentially an identical conclusion about the structure and origin of the so-called rhapidosomes (rod shaped particles) liberated by an even less familiar bacterium, Saprospira grandis, a marine flexibacterium. The elegant electron micrographs of these authors clearly reveal that rhapidosomes are in essence two overlapping hollow cylinders. The core cylinder is some $4,300 \AA$ long and $90 \AA$ in diameter and the sheath cylinder is $2,000 \AA$ long, 250 $\AA$ in diameter and has a channel $110 \AA$ in diameter. Amino-acid analyses of core and sheath prove that the two components are made of distinct protein subunits and there is no nucleic acid associated with the rhapidosome. Delk and Dekker attribute earlier claims that these structures contain methylated RNA to contamination of the rhapidosomes with cellular RNA.

Both the chemical composition and the structure of the core and sheath are so reminiscent of bacteriophage tails

\title{
Sco X-1 af Optical and X-ray Frequencies
}

IT is vital for an understanding of the X-ray source Sco X-1 that it should be determined unambiguously whether or not the optical activity of the source is related to the $\mathrm{X}$-ray variations. Some recent work has found an inverse correlation between optical luminosity and intensity of hard X-rays (T. Kitamura et al., Astrophys. Space Sci., 12, 378 ; 1971); other reports suggest a coincidence between optical and X-ray activity (H. S. Hudson et al., Astrophys. J. Lett., 159, L51; 1970). Part of the problem of resolving this discrepancy, of course, lies in the difficulty of timing balloon or rocket fights to coincide with optical observations.

In next Monday's Nature Physical Science (March 27), M. Matsuoka et al. report further simultaneous observations of this source at optical and X-ray frequencies, and this work supports the evidence for a positive correlation between optical and thermal X-ray emissions. It is also interesting that the X-ray spectra of Sco X-1 show a possible non-thermal component at energies above $50 \mathrm{keV}$ (see figure) which may be enhanced when the source is optically bright. Although this work goes some way towards ex- plaining previous contradictory results, further observations will be required before the nature of the energy generation mechanism becomes clear.

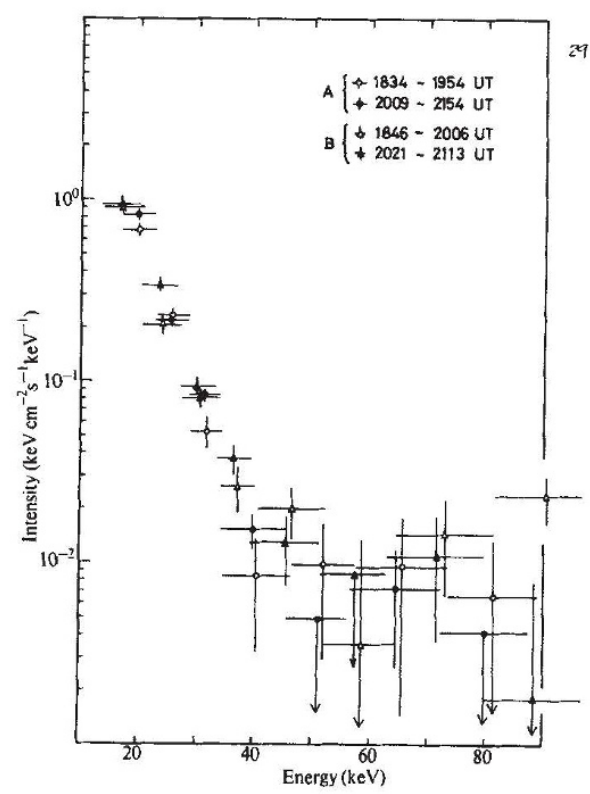

Spectrum of Sco X-1. Circles and triangles represent intensities obtained with two different counters on the same rocket flight on May 1, 1971. 